\title{
Epigenetic mechanisms in pulmonary arterial hypertension: the need for global perspectives
}

\author{
Prakash Chelladurai ${ }^{1}$, Werner Seeger ${ }^{1,2}$ and Soni Savai Pullamsetti ${ }^{1,2}$
}

Affiliations: ${ }^{1}$ Max-Planck-Institute for Heart and Lung Research, Dept of Lung Development and Remodeling, member of the German Center for Lung Research (DZL), Bad Nauheim, Germany. ${ }^{2}$ University of Giessen Lung Center (UGMLC), member of the German Center for Lung Research (DZL), Justus-Liebig University, Giessen, Germany.

Correspondence: Soni Savai Pullamsetti, Max-Planck-Institute for Heart and Lung Research, Dept of Lung Development and Remodeling, Parkstrasse-1, 61231-Bad Nauheim, Germany.

E-mail: soni.pullamsettiampi-bn.mpg.de

ABSTRACT Pulmonary arterial hypertension (PAH) is a severe and progressive disease, characterised by high pulmonary artery pressure that usually culminates in right heart failure. Recent findings of alterations in the DNA methylation state of superoxide dismutase 2 and granulysin gene loci; histone $\mathrm{H} 1$ levels; aberrant expression levels of histone deacetylases and bromodomain-containing protein 4; and dysregulated microRNA networks together suggest the involvement of epigenetics in PAH pathogenesis. Thus, PAH pathogenesis evidently involves the interplay of a predisposed genetic background, epigenetic state and injurious events. Profiling the genome-wide alterations in the epigenetic mechanisms, such as DNA methylation or histone modification pattern in PAH vascular cells, may explain the great variability in susceptibility and disease severity that is frequently associated with pronounced remodelling and worse clinical outcome. Moreover, the influence of genetic predisposition and the acquisition of epigenetic alterations in response to environmental cues in PAH progression and establishment has largely been unexplored on a genome-wide scale. In order to gain insights into the molecular mechanisms leading to the development of $\mathrm{PAH}$ and to design novel therapeutic strategies, high-throughput approaches have to be adopted to facilitate systematic identification of the disease-specific networks using next-generation sequencing technologies, the application of these technologies in PAH has been relatively trivial to date.

@ERSpublications

An epigenetic component is hypothesised in PAH: an overview of the current literature and future perspectives http://ow.ly/7miS3002BYw

\section{Introduction}

Pulmonary hypertension $(\mathrm{PH})$ is a complex and multifactorial cardiopulmonary disorder characterised by a sustained increase in mean pulmonary arterial pressure (mPAP), leading to right ventricular (RV) failure and premature death [1]. Pulmonary arterial hypertension (PAH) describes a subpopulation of patients with $\mathrm{PH}$ that are characterised haemodynamically by the presence of pre-capillary PH. Idiopathic PAH (IPAH) is a sporadic disease and one of the most common forms of PAH, with neither a family history of PAH nor an identified risk factor [2]. Germline mutations in the gene encoding bone morphogenetic protein receptor type-2 (BMPR2) are a major predisposing genetic factor among heritable PAH (HPAH) cases, which segregate in an autosomal dominant pattern, with markedly reduced penetrance [2-4]. Several new loci

Editorial comments in Eur Respir Rev 2016; 25: 104-107.

Received: April 192016 | Accepted after revision: May 062016

Conflict of interest: None declared.

Provenance: Submitted article, peer reviewed.

Copyright OERS 2016. ERR articles are open access and distributed under the terms of the Creative Commons Attribution Non-Commercial Licence 4.0. 
harbouring mutations among patients with and without familial PAH were recently identified through genome-wide association studies and whole-exome sequencing [2, 3]. HPAH is characterised by reduced penetrance, variable expressivity and female predominance, suggesting a crucial contribution of both genetic and environmental modifiers in altering PAH phenotype expression [4].

\section{Epigenetics}

Epigenetics is generally defined as heritable changes in gene activity and expression that occur without alteration in DNA sequence [5]. Epigenetic modifications can be acquired de novo or may be inherited and represent the way in which the genome and environment interact [6]. Epigenetic modifications play a key role in the establishment of a cell-type specific gene expression pattern that is fundamental for lineage commitment, differentiation and proliferation of cells by altering chromatin organisation and consequent gene expression [7]. Changes in the chromatin state of specific genes can lead to their repression or activation. Regulation of chromatin structure and gene expression involves several interconnected mechanisms including: 1) DNA methylation, 2) ATP-dependent chromatin remodelling, 3) histone post-translational modifications (PTMs), 4) noncoding RNAs, 5) replacement of canonical histones with histone variants, and 6) organisation within the three-dimensional nuclear architecture [8].

\section{Role of epigenetic mechanisms in $\mathrm{PAH}$}

Although concrete data are still lacking to support the current hypothesis that an epigenetic component is largely involved during the initiation, progression and establishment phases of $\mathrm{PAH}$, recent findings of alterations in DNA methylation levels at superoxide dismutase 2 (SOD2) and granulysin (GNLY) gene loci; histone H1 levels; aberrant expression levels of histone deacetylases (HDACs) and bromodomain-containing protein 4 (BRD4); and dysregulated microRNA (miRNA) networks have together strengthened the concept of an "epigenetic hypothesis". Notably, we and others have observed that vascular cells (pulmonary artery endothelial cell (PAEC), smooth muscle cell (PASMC) and adventitial fibroblasts) isolated from hypertensive lung vessels and cultured ex vivo outside their vascular microenvironment maintain a hyper-proliferative, apoptosis-resistant phenotype for a longer time than control cells [9-11], demonstrating the presence of a heritable and sustained phenotype. This review provides a comprehensive overview of DNA methylation, histones and histone deacetylases, whereas an overview of miRNA regulation in $\mathrm{PH}$ can be found in the following reviews $[12,13]$.

\section{DNA methylation}

DNA methylation is perhaps the best characterised chemical modification of chromatin that is linked to transcriptional silencing, and is important for gene regulation, development and tumorigenesis [14]. Regions of the genome that have a high density of CpG sites are referred to as CpG islands, and DNA hypermethylation of these islands correlates with transcriptional repression [15]. In PAH, SOD2 expression was found to be diminished in the PASMCs isolated from fawn-hooded rats and plexiform lesions of PAH [16, 17]. ARCHER et al. [17] demonstrated that the selective hypermethylation of CpG islands in the promoter and intronic regions of the SOD2 gene reduces its expression by $\sim 50 \%$ compared with PASMCs from genetically matched consomic rats. Recently, Perros et al. [18] demonstrated the presence of differential epigenetic regulation via hypermethylation of the GNLY gene in explanted lungs and peripheral blood mononuclear cells specifically in pulmonary veno-occlusive disease (PVOD), but not in IPAH or HPAH. In addition, PVOD was specifically associated with a decrease in the cytotoxic T- and natural killer (NK) T-cell subpopulations and an increase in NK cell populations. Interestingly, despite the reduced number of GNLY-containing cells in patients with PVOD, the GNLY concentration in the serum of PVOD patients was significantly higher than in patients with PAH. These important findings may not only contribute to fundamental understanding of the pathomechanisms involving alterations in the circulating and pulmonary cytolytic compartment of the inflammatory cells of PVOD and PAH, but also allows diagnosis and discrimination between patients with PVOD and PAH [18].

\section{Histone post-translational modifications}

Histone PTMs constitute the so-called "histone code" and are read and recognised by reader proteins to orchestrate a variety of chromatin-associated processes such as transcriptional regulation, chromatin condensation, genetic imprinting or DNA damage repair [19]. Lysine acetylation of core histones is one of the best-characterised PTMs that is tightly regulated by the opposing actions of histone acetyltransferases (HATs) and HDACs.

\section{Deregulation of HDAC isoforms and therapeutic evaluation}

The role of aberrant HDAC activity has been strongly implicated in PAH pathogenesis based on the promising therapeutic benefits observed upon the application of small molecule HDAC inhibitors in different animal models of $\mathrm{PH}$ in nine different studies. However, only three studies have reported 
evidence of aberrant gene or protein expression of HDAC isoforms in pulmonary tissues, including human lung homogenates (HDAC1, HDAC4 and HDAC5) [20], human PAH-PAECs (HDAC4 and HDAC5) [21] and from pulmonary adventitial fibroblasts from chronically hypoxic calves (HDAC1, HDAC2 and HDAC3) [9]. However, the pulmonary vascular cell-specific expression and molecular function of specific HDAC isoforms in health and disease remains to be thoroughly explored in $\mathrm{PH}$.

HDAC inhibitors are in use for the treatment of cancer on the basis of their anti-proliferative and pro-apoptotic effects on transformed cells. In addition, preclinical studies have revealed that pan-HDAC inhibitors potently suppress inflammation, fibrosis and restenosis, and have shown efficacy in animal models of left ventricular (LV) dysfunction, which suggests unforeseen potential for HDAC inhibitors for the treatment of $\mathrm{PH}$ and $\mathrm{RV}$ failure [22].

Li et al. [9] were the first to report that inhibition of class I HDACs using apicidin significantly suppressed production of pro-inflammatory mediators by $\mathrm{PH}$-fibroblasts. ZHAO et al. [20] evaluated the therapeutic potential of the broad-spectrum HDAC inhibitors valproic acid (VPA), which inhibits class I HDACs, and suberoylanilide hydroxamic acid (SAHA), which inhibits class I and II HDACs, in rodent models of PH. Both SAHA and VPA mitigated the development of $\mathrm{PH}$ and reduced established hypoxia-induced $\mathrm{PH}$ in rats. Along similar lines, CAVASIN et al. [22] specifically investigated the class I HDAC inhibitors MGCD0103 (Mocetinostat) or MS-275 (Entinostat), which significantly reduced PAP and maintained RV function in MGCD0103-treated animals. These findings further suggest that class-selective HDAC inhibitors will be well tolerated in $\mathrm{PH}$.

Recently, KIM et al. [21] demonstrated that myocyte enhancer factor 2 transcriptional activity was impaired in PAH PAECs mediated by the excess nuclear accumulation of HDAC4 and HDAC5. Selective, pharmacological inhibition of class IIa HDACs using MC1568 restored myocyte enhancer factor 2 activity in IPAH PAECs and rescued from experimental PH (monocrotaline (MCT) and SU5416 plus hypoxia) with no RV fibrosis or coronary artery endothelial cell apoptosis [21]. However, a recent study reported that the class IIa HDAC inhibitor MC1568 moderately suppressed class IIb HDACs, but failed to inhibit class IIa HDAC catalytic activity on a class-selective synthetic substrate in rat left ventricular homogenates [23], while other studies report pan-inhibition of both class IIa and class IIb HDAC isoforms by MC1568 employing recombinant proteins on histones [24].

\section{Mixed results of HDAC inhibition in the right ventricle}

Few studies have reported the beneficial effects of small molecule inhibitors of class I HDACs in preclinical models of LV and RV failure, blocking pathological cardiac hypertrophy and fibrosis and improving ventricular function [25]. Contrary to initial findings of the beneficial effects of HDAC inhibition using VPA on pulmonary artery banding (PAB) or MCT-induced cardiac hypertrophy [26] and trichostatin A (TSA) on transverse aortic constriction-induced cardiac hypertrophy [27], BOGAARD et al. [28] reported detrimental effects of TSA and VPA treatment in PAB-induced RV hypertrophy. A second study by the same group (in 2014) employing three different rodent models (MCT, SU5416 plus hypoxia and PAB) reported no beneficial effects of TSA in SU5416 plus hypoxia-induced PH [29]. These reports cast doubt on the utility of this class of compounds for the treatment of PAH-associated RV dysfunction.

However, the promising therapeutic effects of class-specific HDAC inhibitors in other studies highlight the need for identification of deregulated isoforms in the PAH setting, in order to avoid the detrimental effects of pan-HDAC inhibition observed with drugs like TSA.

\section{Bromodomain-containing proteins and therapeutic evaluation}

Histone acetylation is a key mechanism that regulates chromatin structure and provides docking sites for bromodomains. BRD4 is a member of the BET (bromodomain and extra terminal domain) family that recognise acetylated lysine residues [30]. Recently, MELOCHE et al. [11] reported upregulation of BRD4 in the lungs, distal pulmonary arteries and PASMCs of patients with PAH compared with controls, and confirmed it to be miR-204 dependent. Pharmacological inhibition of BRD4 reversed SU5416 plus hypoxia-induced PAH in vivo, thus confirming that BRD4 upregulation is pathologically associated with $\mathrm{PAH}$ and is a valuable therapeutic target [11]. It is also important to consider that the histone acetylation state in PAH vascular cells is predictably altered due to the dysregulation of HDAC isoforms.

Moreover, significant increases in two fragments of linker histone H1 were identified in the pulmonary arteries from the explanted lungs of IPAH patients compared with controls. However, a reduction in the nuclear histone $\mathrm{H} 1$ and an increase in the cytoplasmic $\mathrm{H} 1$ were observed in IPAH cells. The decreased nuclear $\mathrm{H} 1$ contributes the less compact nucleosomal pattern in IPAH and thus contributes to the increase in nucleosomal repeat length and ultimately to changes in transcription [31]. 


\section{Concluding remarks}

Despite our progressive understanding of the aetiology, pathology and pathogenesis of $\mathrm{PAH}$, and the achievement of mortality reduction in $\mathrm{PAH}$ patients treated with targeted therapies, $\mathrm{PAH}$ still remains an incurable disease [32]. Although the genetic mechanisms and cell signalling abnormalities involved in $\mathrm{PAH}$ pathogenesis have been well studied, our current evidence also highlights that PAH is a complex disease that is mediated by the interplay of predisposed genetic background, epigenetic state and injurious events (figure 1). As discussed in this review, recent evidence points to the involvement of epigenetic changes in the pathogenesis of PAH. Consistent with the observations of stable disease phenotype exhibited by transformed cells in hyper-proliferative diseases such as cancer, ex vivo cultured PAH pulmonary vascular cells exhibit stable increases in cell proliferation, resistance to apoptosis, metabolic switch and pro-inflammatory gene expression. This hints at the presence of disease favourable alterations in the gene regulatory signatures that facilitate the normal pulmonary vascular cells to acquire and consistently exhibit stable pro-proliferative, anti-apoptotic, pro-inflammatory and pro-fibrotic vascular cell phenotypes, thereby aggravating the vascular remodelling process. The epigenetic hypothesis gives credit to the impact of the environment and the consequent epigenetic alterations as a contributing factor for $\mathrm{PAH}$.

In order to improve the current understanding of $\mathrm{PAH}$ pathogenesis, a considerable shift in our efforts are desired, largely towards the identification of epigenetic mechanisms involved in the establishment of PAH. This can be facilitated by the application of next-generation sequencing (NGS) technologies, which have the potential to substantially accelerate epigenomic research in the PAH setting. The spectrum of applications for NGS-based profiling includes genome-wide analysis of DNA methylation patterns, post-translational modifications of histones and the coding and non-coding transcriptome, on a genome-wide scale. The impact of epigenetic changes such as DNA methylation and histone modification has been better documented in the pathogenesis of other human diseases [33-36], while the application of genomic technologies in human PAH samples are relatively limited. To date, except for a few studies [37-40], the approaches employed to delineate

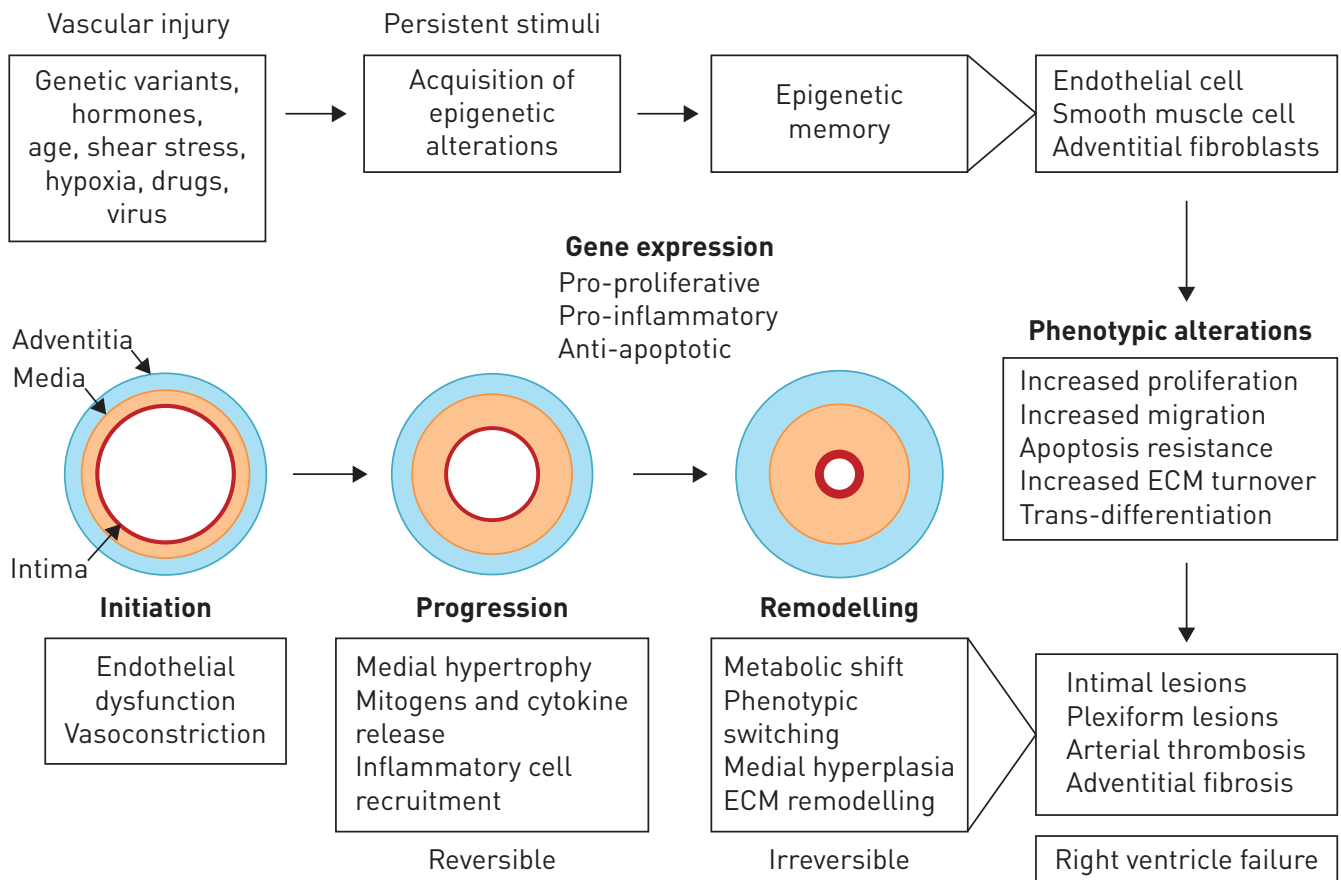

FIGURE 1 Epigenetic mechanisms in pulmonary arterial hypertension (PAH). Genetic predisposition, age, sex, environmental factors and pathological stimuli such as hypoxia, shear stress, oxidative stress, infection, mitogens and inflammation (cytokines and chemokines) cause vascular injury. Endothelial dysfunction causes an imbalance in the production of several vasoactive substances thereby facilitating vasoconstriction. Activation of downstream signalling cascades arbitrates PAH progression via recruitment and modulation of transcription factors that regulate stimulus-specific transcriptional responses. Following the persistence of pathological stimuli, the gene regulatory signatures in the normal vascular cells are altered and thereby establish a phenotypic memory in PAH vascular cells. Aside from the hypoxia-induced metabolic shift, acquisition of epigenetic alterations consequently facilitates stimulus-independent aberrant transcription of pro-proliferative, pro-migratory, anti-apoptotic, pro-inflammatory and pro-fibrotic genes that underlie the different vascular cell phenotypes in PAH vasculature. Phenotypic switching of pulmonary vascular cells largely contributes to the development of complex vascular lesions during the progressive vascular remodelling process in $\mathrm{PAH}$. ECM: extracellular matrix. 
the disease mechanisms in PAH have not provided a global perspective of the molecular network underlying the disease phenotype. To gain insight into the mechanisms leading to the development of PAH, a broad range of high-throughput techniques in genomics, proteomics and metabolomics can be employed to systematically dissect the disease-specific networks in isolated pulmonary vascular cells, peripheral blood mononuclear cells and pulmonary tissues collected from patients.

Although the spectrum of therapeutic options for PAH has expanded in past 20 years, the targeted therapies that are approved and available for treatment of PAH largely result from the understanding of the central role of nitric oxide, nitric oxide-cGMP, prostacyclin and endothelin pathways in PAH pathogenesis [32, 41]. Several research groups are currently evaluating the therapeutic efficacy of modulation of HDACs in PAH pathogenesis using small-molecule inhibitors. In essence, there is a need to identify specific isoforms that are deregulated in the PAH pulmonary vasculature and for application of isoform-selective inhibitors to largely minimise the off-target effects experienced upon broad-spectrum inhibition. Analysis of the epigenome will be a decisive factor in identification of disease-specific regulatory networks that aggravate the disease, since the epigenetic state of a cell determines cellular phenotype during developmental or disease. It is therefore crucial to profile the vascular cell-specific epigenome that will not only provide key insights into the pathogenesis of $\mathrm{PAH}$, but can also be employed as biomarkers for clinical diagnosis and prognosis. Furthermore, the "integromics" perspective will also be necessary to comprehensively elucidate the disease-specific network involving genetic, epigenetic and transcription factors that may direct the development of novel therapeutic strategies for achieving reverse remodelling in $\mathrm{PAH}$, in near future.

\section{References}

1 Hoeper MM, Bogaard HJ, Condliffe R, et al. Definitions and diagnosis of pulmonary hypertension. J Am Coll Cardiol 2013; 62: Suppl., D42-D50.

2 Montani D, Günther S, Dorfmüller P, et al. Pulmonary arterial hypertension. Orphanet J Rare Dis 2013; 8: 97.

3 Soubrier F, Chung WK, Machado R, et al. Genetics and genomics of pulmonary arterial hypertension. J Am Coll Cardiol 2013; 62: Suppl., D13-D21.

4 Austin ED, Loyd JE. Heritable forms of pulmonary arterial hypertension. Semin Respir Crit Care Med 2013; 34: 568-580.

5 Goldberg AD, Allis CD, Bernstein E. Epigenetics: a landscape takes shape. Cell 2007; 128: 635-638.

6 Liu L, Li Y, Tollefsbol TO. Gene-environment interactions and epigenetic basis of human diseases. Curr Issues Mol Biol 2008; 10: 25-36.

7 Trerotola M, Relli V, Simeone P, et al. Epigenetic inheritance and the missing heritability. Hum Genomics 2015; 9: 17.

8 Bönisch C, Hake SB. Histone H2A variants in nucleosomes and chromatin: more or less stable? Nucleic Acids Res 2012; 40: 10719-10741.

9 Li M, Riddle SR, Frid MG, et al. Emergence of fibroblasts with a proinflammatory epigenetically altered phenotype in severe hypoxic pulmonary hypertension. J Immunol 2011; 187: 2711-2722.

10 Masri FA, Xu W, Comhair SA, et al. Hyperproliferative apoptosis-resistant endothelial cells in idiopathic pulmonary arterial hypertension. Am J Physiol Lung Cell Mol Physiol 2007; 293: L548-L554.

11 Meloche J, Potus F, Vaillancourt M, et al. Bromodomain-containing protein 4: the epigenetic origin of pulmonary arterial hypertension. Circ Res 2015; 117: 525-535.

12 Zhou G, Chen T, Raj JU. MicroRNAs in pulmonary arterial hypertension. Am J Respir Cell Mol Biol 2015; 52: 139-151.

13 Kim JD, Lee A, Choi J, et al. Epigenetic modulation as a therapeutic approach for pulmonary arterial hypertension. Exp Mol Med 2015; 47: e175.

14 Ehrlich M. DNA hypomethylation in cancer cells. Epigenomics 2009; 1: 239-259.

15 Gao F, Liang H, Lu H, et al. Global analysis of DNA methylation in hepatocellular carcinoma by a liquid hybridization capture-based bisulfite sequencing approach. Clin Epigenetics 2015; 7: 86.

16 Kim GH, Ryan JJ, Marsboom G, et al. Epigenetic mechanisms of pulmonary hypertension. Pulm Circ 2011; 1: 347-356.

17 Archer SL, Marsboom G, Kim GH, et al. Epigenetic attenuation of mitochondrial superoxide dismutase 2 in pulmonary arterial hypertension: a basis for excessive cell proliferation and a new therapeutic target. Circulation 2010; 121: 2661-2671.

18 Perros F, Cohen-Kaminsky S, Gambaryan N, et al. Cytotoxic cells and granulysin in pulmonary arterial hypertension and pulmonary veno-occlusive disease. Am J Respir Crit Care Med 2013; 187: 189-196.

19 Cohen I, Poręba E, Kamieniarz K, et al. Histone modifiers in cancer: friends or foes? Genes Cancer 2011; 2 : 631-647.

20 Zhao L, Chen $\mathrm{CN}$, Hajji $\mathrm{N}$, et al. Histone deacetylation inhibition in pulmonary hypertension: therapeutic potential of valproic acid and suberoylanilide hydroxamic acid. Circulation 2012; 126: 455-467.

21 Kim J, Hwangbo C, Hu X, et al. Restoration of impaired endothelial myocyte enhancer factor 2 function rescues pulmonary arterial hypertension. Circulation 2015; 131: 190-199.

22 Cavasin MA, Demos-Davies K, Horn TR, et al. Selective class I histone deacetylase inhibition suppresses hypoxia-induced cardiopulmonary remodeling through an antiproliferative mechanism. Circ Res 2012; 110: 739-748.

23 Lemon DD, Harrison BC, Horn TR, et al. Promiscuous actions of small molecule inhibitors of the protein kinase D-class IIa HDAC axis in striated muscle. FEBS Lett 2015; 589: 1080-1088.

24 Nebbioso A, Manzo F, Miceli M, et al. Selective class II HDAC inhibitors impair myogenesis by modulating the stability and activity of HDAC-MEF2 complexes. EMBO Rep 2009; 10: 776-782.

25 Cavasin MA, Stenmark KR, McKinsey TA. Emerging roles for histone deacetylases in pulmonary hypertension and right ventricular remodeling (2013 Grover Conference series). Pulm Circ 2015; 5: 63-72. 
Cho YK, Eom GH, Kee HJ, et al. Sodium valproate, a histone deacetylase inhibitor, but not captopril, prevents right ventricular hypertrophy in rats. Circ J 2010; 74: 760-770.

27 Kong Y, Tannous P, Lu G, et al. Suppression of class I and II histone deacetylases blunts pressure-overload cardiac hypertrophy. Circulation 2006; 113: 2579-2588.

28 Bogaard HJ, Mizuno S, Hussaini AA, et al. Suppression of histone deacetylases worsens right ventricular dysfunction after pulmonary artery banding in rats. Am J Respir Crit Care Med 2011; 183: 1402-1410.

29 De Raaf MA, Hussaini AA, Gomez-Arroyo J, et al. Histone deacetylase inhibition with trichostatin A does not reverse severe angioproliferative pulmonary hypertension in rats (2013 Grover Conference series). Pulm Circ 2014; 4: $237-243$.

30 Filippakopoulos P, Knapp S. Targeting bromodomains: epigenetic readers of lysine acetylation. Nat Rev Drug Discov 2014; 13: 337-356.

31 Talati M, Seeley E, Ihida-Stansbury K, et al. Altered expression of nuclear and cytoplasmic histone H1 in pulmonary artery and pulmonary artery smooth muscle cells in patients with IPAH. Pulm Circ 2012; 2: 340-351.

32 Galiè N, Manes A, Negro L, et al. A meta-analysis of randomized controlled trials in pulmonary arterial hypertension. Eur Heart J 2009; 30: 394-403.

33 Irizarry RA, Ladd-Acosta C, Wen B, et al. The human colon cancer methylome shows similar hypo- and hypermethylation at conserved tissue-specific CpG island shores. Nat Genet 2009; 41: 178-186.

34 Sharma P, Garg G, Kumar A, et al. Genome wide DNA methylation profiling for epigenetic alteration in coronary artery disease patients. Gene 2014; 541: 31-40.

35 Simmer F, Brinkman AB, Assenov Y, et al. Comparative genome-wide DNA methylation analysis of colorectal tumor and matched normal tissues. Epigenetics 2012; 7: 1355-1367.

36 Rabinovich EI, Kapetanaki MG, Steinfeld I, et al. Global methylation patterns in idiopathic pulmonary fibrosis. PLoS One 2012; 7: e33770.

37 Bertero T, Lu Y, Annis S, et al. Systems-level regulation of microRNA networks by miR-130/301 promotes pulmonary hypertension. J Clin Invest 2014; 124: 3514-3528.

38 Rajkumar R, Konishi K, Richards TJ, et al. Genomewide RNA expression profiling in lung identifies distinct signatures in idiopathic pulmonary arterial hypertension and secondary pulmonary hypertension. Am J Physiol Heart Circ Physiol 2010; 298: H1235-H1248.

39 Bull TM, Coldren CD, Moore M, et al. Gene microarray analysis of peripheral blood cells in pulmonary arterial hypertension. Am J Respir Crit Care Med 2004; 170: 911-919.

40 Rhodes CJ, Im H, Cao A, et al. RNA sequencing analysis detection of a novel pathway of endothelial dysfunction in pulmonary arterial hypertension. Am J Respir Crit Care Med 2015; 192: 356-366.

41 Galiè N, Humbert M, Vachiery JL, et al. 2015 ESC/ERS Guidelines for the diagnosis and treatment of pulmonary hypertension. Eur Heart J 2016; 37: 67-119. 